\title{
Preclinical biodistribution and safety study of reduced expression in immortalized cells/Dickkopf-3-encoding adenoviral vector for prostate cancer gene therapy
}

\author{
MORITO SUGIMOTO ${ }^{1}$, MASAMI WATANABE ${ }^{1-3}$, HARUKI KAKU $^{1-3}$, SHUN-AI LI $^{2,3}$, HIROFUMI NOGUCHI $^{4}$, \\ HIDEO UEKI $^{1}$, MASAKIYO SAKAGUCHI ${ }^{5}$, NAM-HO $\mathrm{HUH}^{5}$, YASUTOMO NASU ${ }^{1-3}$ and HIROMI KUMON ${ }^{1-3}$ \\ ${ }^{1}$ Department of Urology, ${ }^{2}$ Center for Gene and Cell Therapy, ${ }^{3}$ Innovation Center Okayama for Nanobio-Targeted Therapy, \\ Departments of ${ }^{4}$ Gastroenterological Surgery and ${ }^{5}$ Cell Biology, Graduate School of Medicine, Dentistry, \\ and Pharmaceutical Sciences, Okayama University, Okayama 700-8558, Japan
}

Received April 9, 2012; Accepted July 9, 2012

DOI: 10.3892/or.2012.2001

\begin{abstract}
The biodistribution and safety of adenoviral vectors encoding the human REIC/Dkk-3 tumor suppressor gene (Ad-REIC) were examined in this preclinical study for in situ prostate cancer gene therapy. First, the in vitro apoptotic effects of Ad-REIC in normal and cancer cells derived from the prostate and liver were examined. Significant apoptotic effects were observed at $100 \mathrm{MOI}$ (multiplicity of infection) in prostate cancer cells (LNCaP, PC3) and hepatoma cells (HEP3B and HEPG2); however, no effects were seen in normal cells. To analyze the safety of intraprostatic Ad-REIC administration, the biodistribution and histology after Ad-REIC injection were evaluated in various organs of normal male C57BL6 mice. In a supporting study, vector dissemination following intravenous injection of Ad-REIC into tail veins was determined. To evaluate whether Ad-REIC was present in the collected tissue specimens, human REIC gene detection was performed using DNA-PCR. Intraprostatic treatment administered at lower doses showed vector biodistribution into the colon, urinary bladder and prostate. At higher doses, vector dissemination was observed in tissues more distant from the prostate, including the lung, thymus, heart, liver and adrenal gland. After intravenous injection of Ad-REIC, dissemination was observed in the liver and spleen. These results indicate that the biodistribution of Ad-REIC is determined by the dose and route of administration. Although acute inflammatory effects were observed in the prostate after intraprostatic administration at higher doses, no abnormal histological findings were noted in the other tissues, including those of intravenously treated mice. Regarding the safety of Ad-REIC administration, no deaths and no signs
\end{abstract}

Correspondence to: Dr Masami Watanabe, Department of Urology, Okayama University, 2-5-1 Shikata-cho, Okayama 700-8558, Japan E-mail: mwcorrespondence@gmail.com

Key words: biodistribution, adenovirus vector, reduced expression in immortalized cells, gene therapy, prostate cancer of toxicity or unusual behavior were observed in the mice in any treatment group. Based on these preclinical experiments, adenovirus-mediated in situ REIC/Dkk-3 gene therapy is considered to be safe for use as a treatment for human prostate cancer.

\section{Introduction}

Gene therapy has been used to treat human cancers in clinical trials and is considered to have therapeutic potential to treat local-regional cancers. Our group previously cloned the reduced expression in immortalized cells (REIC) gene and reported that its expression is downregulated in a variety of cancer cell lines and tumors (1-5). The REIC gene is identical to the Dickkopf-3 (Dkk-3) gene (1), a member of the Dickkopf (Dkk) gene family known to interfere with Wnt signaling via Wnt-receptors (6). The REIC/Dkk-3 gene has been previously reported to play a distinct role in the induction of apoptosis and the inhibition of metastasis $(7,8)$. The REIC/Dkk-3 gene induces potent antitumor effects in vivo and cancer-specific apoptosis in a variety of cancer types when expressed by adenovirus-mediated gene transfer (9-12). Therefore, REIC/Dkk-3 is one of the most attractive tumor suppressor genes for cancer gene therapy. Based on this background, we are now promoting the transfer of in situ REIC/Dkk-3 gene therapy from the lab to the clinic for use in treating patients with cancer.

The therapeutic effects of adenoviral vectors encoding the REIC/Dkk-3 gene (Ad-REIC) were previously evaluated in a variety of in vivo tumor models using prostate, breast, and mesothelioma cancer cells $(3,4,8-11)$; however, the toxicity of Ad-REIC treatment remains unknown. An important component in the safety evaluation of gene therapy agents is the determination of the biodistribution and dissemination of the agent in vivo following administration by appropriate routes. Because locoregional treatment for prostate cancer is achieved with intraprostatic administration of vectors, adenoviral vectors have been used in several clinical trials of gene therapy (13-15). However, the systemic biodistribution of viral vectors after intraprostatic administration is not fully elucidated. To determine the vector biodistribution of Ad-REIC following 
intraprostatic administration, and as a necessary preclinical study prior to clinical use as a treatment for prostate cancer, we herein examine the biodistribution and safety of intraprostatic injection of Ad-REIC vectors. The use of mice in this kind of preclinical evaluation has many advantages (16); therefore, vector-specific DNA polymerase chain reaction (PCR) was used to track the distribution of the vectors from the prostate to other organs in mice. In a comparative study, we also determined vector distribution following the intravenous systemic administration of Ad-REIC.

\section{Materials and methods}

Cell cultures. Normal human prostate epithelial cells (PrEC) and hepatocytes (HC) were purchased from Lonza (Basel, Switzerland) and cultivated using the medium recommended by the supplier. Human cancer cell lines (LNCaP, PC3, HEP3B, HEPG2) were provided by the American Type Culture Collection (Rockville, MD). Dulbecco's modified Eagle's medium (DMEM; Invitrogen, Carlsbad, CA) and RPMI-1640 medium (Nissui, Tokyo, Japan) were used as the cultures for the cancer cell lines. The cell lines were grown in media supplemented with $10 \%$ fetal bovine serum (FBS; Biowest, Nuaille, France), as previously described (5).

Adenovirus vectors carrying REIC/Dkk-3 (Ad-REIC). The full-length cDNA of the human REIC/Dkk-3 gene was inserted into the cosmid vector, pAxCAwt, and then transferred into an adenoviral vector using the COS-TPC method (Takara Bio, Shiga, Japan) (3). An adenoviral vector carrying the LacZ gene (Ad-LacZ) was used as the control vector. The adenoviral vectors consisted of replication-defective adenovirus of serotype 5 that contained the indicated genes under the control of the CMV early enhancer/chicken $\beta$ actin (CAG) promoter.

Western blot analyses. The total protein of in vitro treated cells and mouse prostatic tissue was extracted with a lysis buffer (50 mM HEPES, pH 7.4, $250 \mathrm{mM} \mathrm{NaCl,} 1 \mathrm{mM}$ EDTA, $1 \%$ NP-40, 1 mM DTT, 1 mM PMSF, $5 \mu \mathrm{g} / \mathrm{ml}$ leupeptin, $5 \mu \mathrm{g} /$ $\mathrm{ml}$ aprotinin, $2 \mathrm{mM} \mathrm{Na} \mathrm{VO}_{4}, 1 \mathrm{mM} \mathrm{NaF}, 10 \mathrm{mM} \beta$-GP). After centrifugation was completed, the volume of the supernatants was adjusted in each sample to achieve equal protein concentrations, and the samples were diluted with the same volume of 2X SDS sample buffer and heated for $5 \mathrm{~min}$ at $95^{\circ} \mathrm{C}$. The samples $(10 \mu \mathrm{g}$ of protein) were then separated on $7.5 \%$ SDS-PAGE gels and electroblotted onto polyvinylidene fluoride (PVDF) membranes. The blots were blocked for $1 \mathrm{~h}$ with $10 \%$ non-fat milk powder, $6 \%$ gycine, and $0.1 \%$ Tween-20 in Tris-buffered saline (TBS) at room temperature. The REIC protein was then identified with the use of a primary antibody: rabbit anti-human REIC/ Dkk-3 polyclonal antibody raised in our laboratory $(3,5)$. After being extensively washed with $0.1 \%$ Tween-20 in TBS (T-TBS), the blots were exposed to horseradish peroxidase-conjugated secondary antibodies. After being extensively washed with T-TBS, the membranes were developed using the enhanced chemiluminescence detection method (ECL kit, Amersham Pharmacia Biotech, Chandler, AZ).

Apoptosis assays. To examine the induction of in vitro apoptosis following the treatments, the cells were seeded in flat-bottom 6-well plates and incubated for $24 \mathrm{~h}$. The cells were then treated with either the Ad-CAG-LacZ or Ad-CAG-REIC at the indicated multiplicity of infection (MOI) in the complete medium $(500 \mu \mathrm{l})$ for $1 \mathrm{~h}$. Fresh medium was added to bring the total to $2 \mathrm{ml}$. After an additional $72 \mathrm{~h}$ of incubation, Hoechst 33342 stock solution was added to the medium at a concentration of $2 \mu \mathrm{g} / \mathrm{ml}$ and the cells were incubated in the dark for $10 \mathrm{~min}$. Hoechst 33342 is an intercalating dye that allows a determination of the variation in the total chromatin quantity and the degree of chromatin condensation to be made $(17,18)$. Using fluorescence microscopy, apoptotic cells were identified by the presence of highly condensed or fragmented nuclei. Apoptotic cells were counted in five different fields under microscopic observation, and 100 cells were judged in each field.

Animal experiments. An analysis of the biodistribution of Ad-REIC and a histological evaluation were completed following two doses of vector injection into the dorsolateral prostate (left lobe) and one dose of intravenous injection into the tail vein of each mouse. The treatment groups were classified as follows: control group (group 1), intraprostatic injection of the vehicle [phosphate buffered saline (PBS)]; intraprostatic injection of the vector (group 2), intraprostatic injection of $2.5 \times 10^{8}$ viral particles (vp) of Ad-REIC in $20 \mu \mathrm{l}$ vehicles; intraprostatic injection (group 3), intraprostatic injection of $2.0 \times 10^{10} \mathrm{vp}$ of Ad-REIC in $20 \mu \mathrm{l}$ vehicles; intravenous injection via the tail vein (group 4): intravenous injection of $2.5 \times 10^{8} \mathrm{vp}$ of Ad-REIC in $100 \mu \mathrm{l}$ vehicles. A total of 27 mice (C57BL/6 male mice, 6-8 weeks of age) were treated in order to obtain 19 evaluable animals, one for the control and two for each of the Ad-REIC treatment groups for each time-point. The first day of vector administration was designated as day 0 , and the mice were sacrificed on days 1,7 and 28. The physical appearance, activity levels, and mortality patterns of all of the animals were observed daily. When the mice were sacrificed, the following organs were collected for biodistribution and histological analysis: brain, lung, thymus, heart, liver, pancreas, spleen, kidney, stomach, colon, urinary bladder, dorsolateral prostate (injection site in groups 1-3), testis, femoris muscle, adrenal gland, and whole blood. To complete the DNA-PCR assays of the biodistribution analyses, whole blood was firstly drawn from the inferior vena cava of each animal under deep anesthesia with sodium pentobarbital. The blood samples were collected in a plastic tube and preserved with ethylenediaminetetra-acetic acid (EDTA). The organs were then dissected and stored at $-80^{\circ} \mathrm{C}$. Scalpels and forceps were changed after each organ dissection or incision to avoid cross-contamination of the tissues. To complete the histological analysis of the potential side effects of Ad-REIC treatment, the tissues were fixed in formalin and embedded to create paraffin sections. The sections $(5 \mu \mathrm{m})$ were stained with hematoxylin and eosin and examined for histopathological and cytotoxic changes.

DNA-PCR (polymerase chain reaction) for the analyses of the biodistribution of Ad-REIC. DNA-PCR was performed on each mouse organ to detect the presence of vector DNA derived from the Ad-REIC vectors and to determine the biodistribution of Ad-REIC. The organs were weighed and each ( 25 mg) was suspended in $180 \mu \mathrm{l}$ of ATL buffer (QIAamp DNA Mini kit, Qiagen) with $20 \mu \mathrm{l}$ of proteinase K. A $200-\mu 1$ aliquot of each organ was incubated at $56^{\circ} \mathrm{C}$ until the tissue was completely 
A

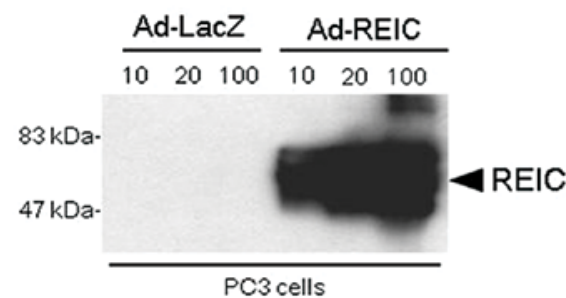

B

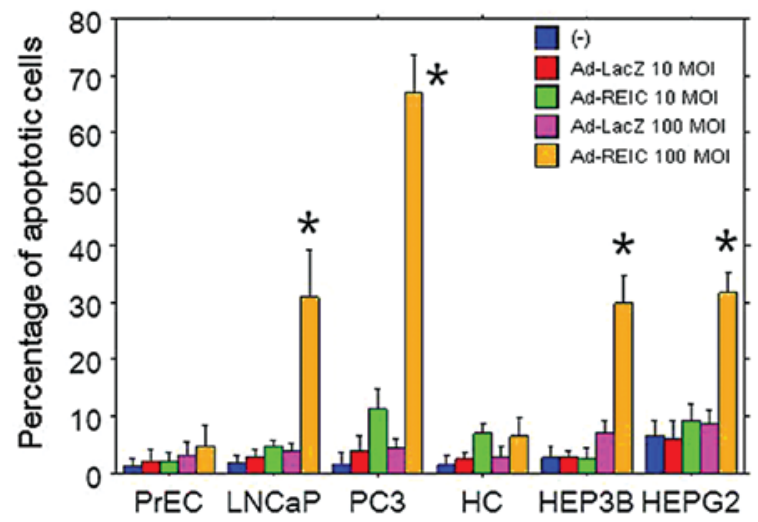

(-) $\frac{A d-L C^{2} C^{2}}{100} \frac{A d-R E I C}{100}$

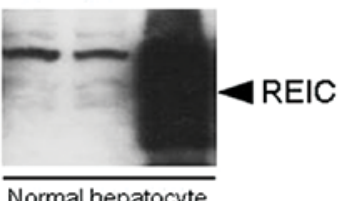

C

Ad-REIC (100 MOI)

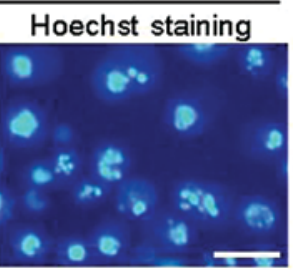

Bright field

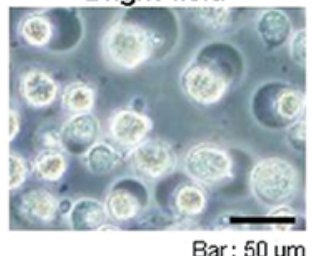

Figure 1. (A) REIC/Dkk-3 expression following in vitro Ad-REIC treatment was examined in PC3 human prostate cancer cells and normal human hepatocytes using western blot analysis. Total protein was extracted on day 1 after the treatment and $10 \mu \mathrm{g}$ of protein were loaded in each lane. (B) The rates of apoptosis in various human cancer cells (prostate cancer, LNCaP, PC3; hepatoma, HEP3B, HEPG2) and normal human cells (prostate epithelial cells, PrEC; hepatocytes, HC) following the indicated treatments. A total of five different fields were examined under a microscope to determine the rates of apoptosis. A significant difference was observed $\left({ }^{*} \mathrm{p}<0.05\right)$ between the Ad-REIC $(100 \mathrm{MOI})$ treatment group and the control Ad-LacZ (100 MOI) treatment group. $(\mathrm{C})$ The appearance of apoptotic cells in PC3 cancer cells following the Ad-REIC treatment is shown using Hoechst 33342 staining.

lysed and then was placed at room temperature. AL buffer (200 $\mu \mathrm{l}$ ) was added to each sample and the samples were incubated at $70^{\circ} \mathrm{C}$ for $10 \mathrm{~min} .100 \%$ ethanol $(200 \mu \mathrm{l})$ was added to each sample and the samples were mixed thoroughly and then placed into QIAamp spin-columns. After several washing steps were completed, the DNA was eluted with $200 \mu$ l of AE buffer. Each DNA sample was used in a PCR that amplified a 621-bp fragment of the REIC gene. The reactions contained $300 \mathrm{ng}$ of sample DNA, 10 pmol of each primer (REIC-F: 5'-ATGCAGCGGCTTGGGGCCACCCTGCT GTGC-3'; REIC-R: 5'-GATGGTCCCATTGCTGCCCCTGGT GGCCAT-3'), 8 mmol of each deoxynucleoside triphosphate, 2X GC buffer, and $1 \mathrm{U}$ of LA Taq (Takara Bio) in a volume of $20 \mu \mathrm{l}$. The reactions were incubated at $98^{\circ} \mathrm{C}$ for $30 \mathrm{sec}$, then 30 cycles of $10 \mathrm{sec}$ at $98^{\circ} \mathrm{C}$ and $30 \mathrm{sec}$ at $72^{\circ} \mathrm{C}$ were performed. Following the final cycle, a 5 -min incubation at $72^{\circ} \mathrm{C}$ was performed. After loading dye was added to the reactions, the samples were electrophoresed in a $1.0 \%$ agarose gel containing $0.5 \mu \mathrm{g} / \mathrm{ml}$ ethidium bromide and visualized with an ultraviolet transilluminator. A sample containing an Ad-REIC vector was assayed as a positive control. A sample without any vector was assayed as a negative control in order to detect any contamination.

Statistical analyses. The data are expressed as the means \pm the standard deviations (SD). An unpaired Student's t-test was used to compare the data between the two groups. Differences were considered to be statistically significant at $\mathrm{p}<0.05$.

\section{Results}

In vitro REIC protein expression and apoptosis induction with Ad-REIC treatment. To confirm whether protein expression and apoptotic effects were induced by Ad-REIC, various human cancer cell lines and normal human cells were treated with Ad-REIC. Western blot analysis demonstrated a significant expression of REIC protein in PC3 human prostate cancer cells and human hepatocytes on day 1 (Fig. 1A). In an apoptosis assay using the Hoechst 33342 stain, significant apoptosis induction was observed in Ad-REIC treated prostate cancer cells (LNCaP, PC3) and hepatoma cells (HEP3B, HEPG2) in comparison to the control Ad-LacZ treated cells; however, no apoptosis was observed in normal cells (PrEC, HC) (Fig. 1B). The mean rate of apoptosis at $100 \mathrm{MOI}$ in the cancer cells (LNCaP, PC3, HEP3B, HEPG2) was 31.0, 67.0, 30.0 and 31.8\%, respectively.

The biodistribution of Ad-REIC in mice after intraprostatic and intravenous injection of the vectors. Human REIC gene detection was performed using DNA-PCR to identify the presence of Ad-REIC vectors in various tissues, including the brain, lung, thymus, heart, liver, pancreas, spleen, kidney, stomach, colon, urinary bladder, prostate (injection site in groups 1-3), testis, femoris muscle, adrenal gland, and whole blood. Total DNA was extracted from each tissue and analyzed for the presence of REIC DNA (621-bp fragment) using PCR. The PCR bands were confirmed with electropho- 

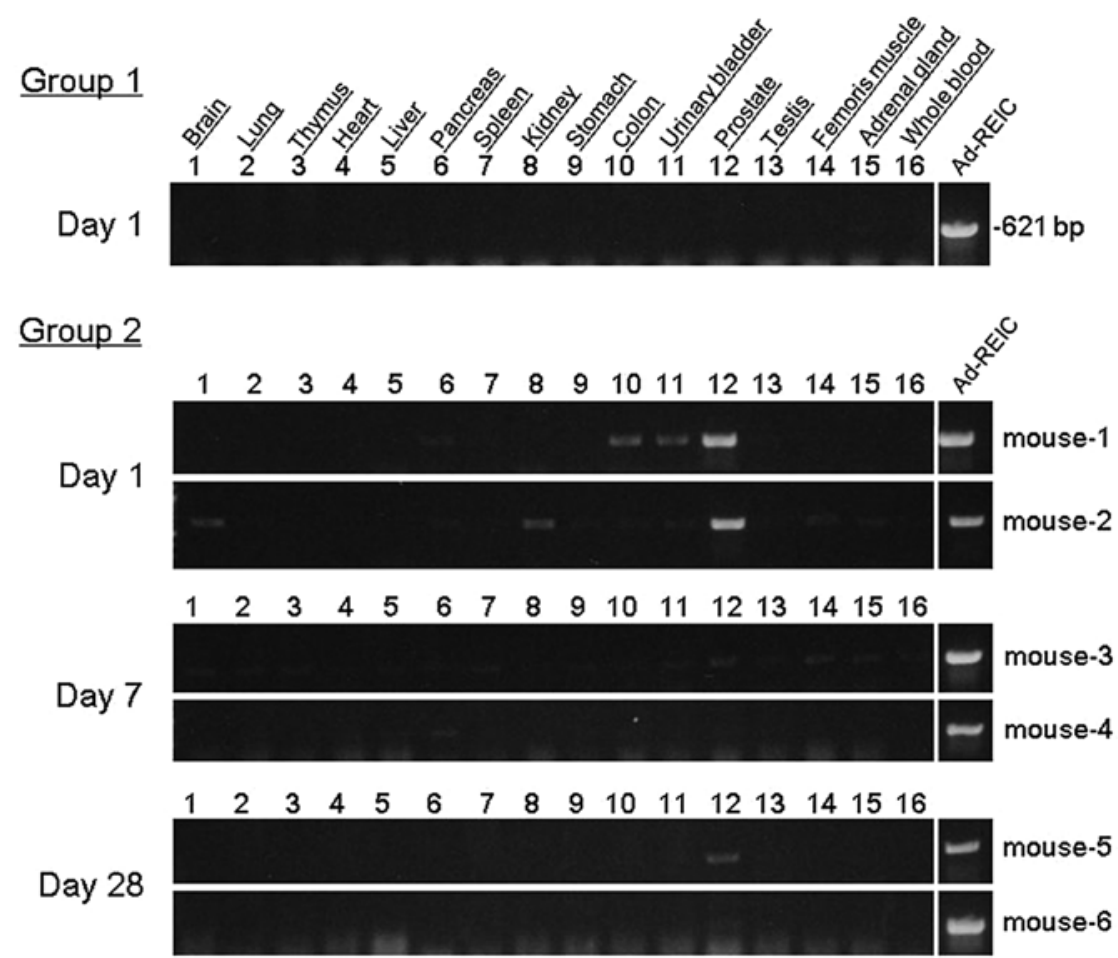

Figure 2. To evaluate the biodistribution of Ad-REIC vectors in the indicated organs, human REIC gene detection was performed using DNA-PCR. Total DNA was extracted from each tissue and analyzed for the presence of REIC DNA (621-bp fragment) using PCR. The PCR bands were detected with electrophoresis on the indicated days following the treatment. The treatment groups were classified as follows: control group (group 1), intraprostatic injection of the vehicle [phosphate buffered saline (PBS)]; intraprostatic injection of the vector (group 2), intraprostatic injection of $2.5 \times 10^{8}$ viral particles (vp) of Ad-REIC in $20 \mu 1$ vehicles.

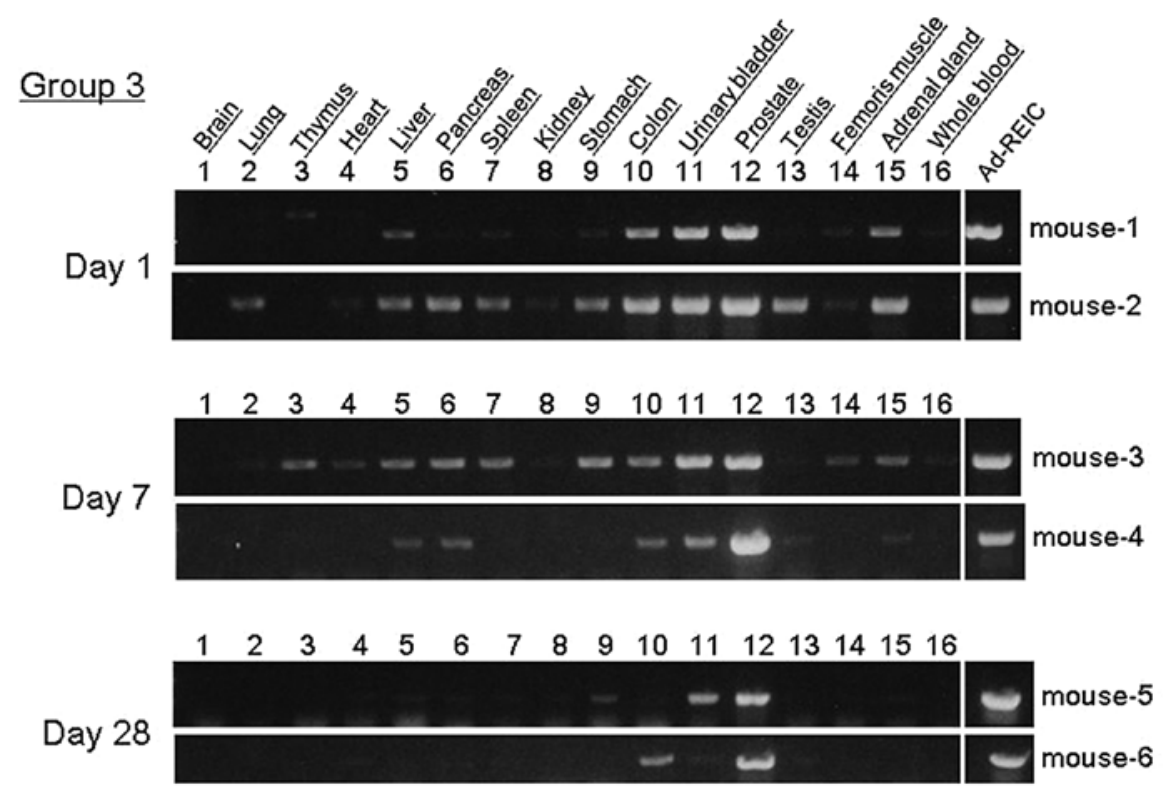

Figure 3. To examine the biodistribution of Ad-REIC vectors in the indicated organs, human REIC gene detection was performed using DNA-PCR. Total DNA was extracted from each tissue and analyzed for the presence of REIC DNA (621-bp fragment) using PCR. The treatment administered in group 3 was as follows: intraprostatic injection of $2.0 \times 10^{10} \mathrm{vp}$ of Ad-REIC in $20 \mu \mathrm{l}$ vehicles.

resis. The analysis used to detect REIC DNA in a given tissue is semi-quantitative and the assay provides a gross indication of the relative distribution of the vector Ad-REIC DNA. The sensitivity of the DNA-PCR was established by adding various amounts of Ad-REIC viral particles into the buffer (ranging from $1 \times 10^{10} \mathrm{vp} / \mathrm{ml}$ to $1 \times 10^{4} \mathrm{vp} / \mathrm{ml}$. The limit of detection for this method was demonstrated to be $1 \times 10^{7} \mathrm{vp} / \mathrm{ml}$; however, trace levels were also visible at $1 \times 10^{6} \mathrm{vp} / \mathrm{ml}$ (data not shown).

In the control group (group 1), no bands of vector DNA derived from Ad-REIC were detected, demonstrating that the assay did not react with the mouse REIC gene and no interference from human REIC DNA occurred in the mice. On day 1 

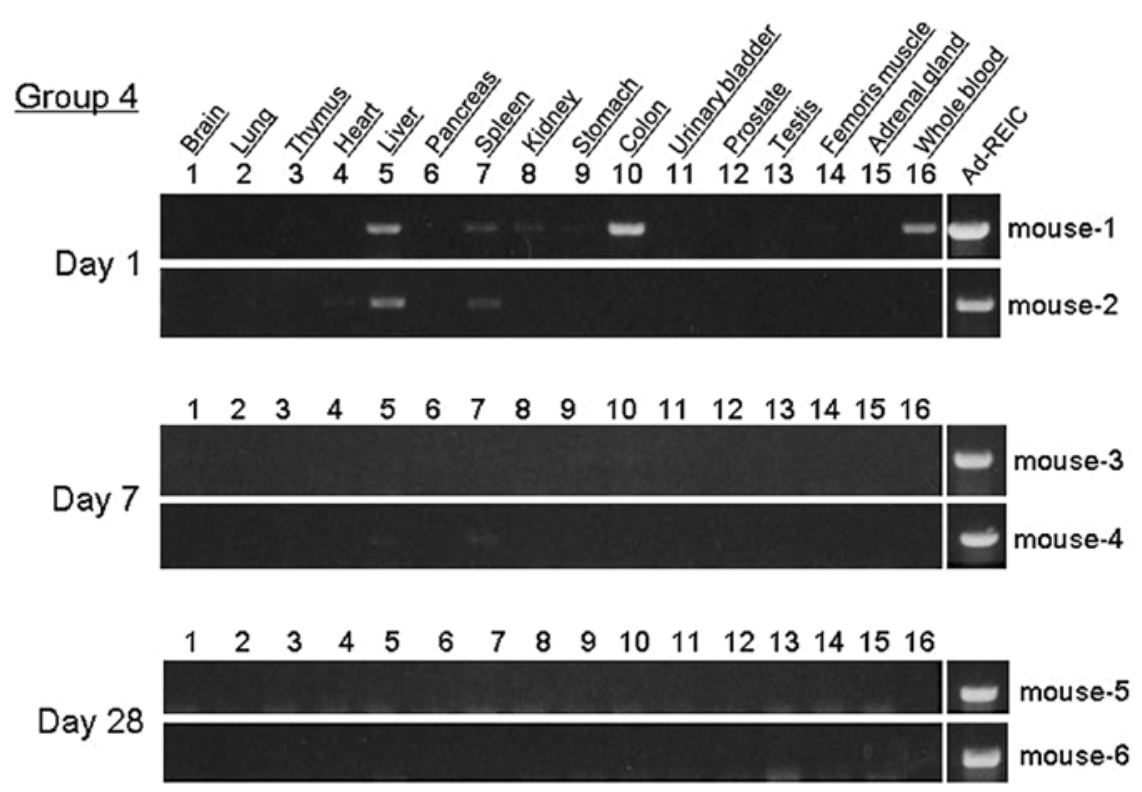

Figure 4. To examine the biodistribution of Ad-REIC vectors in the indicated organs, human REIC gene detection was performed using DNA-PCR. Total DNA was extracted from each tissue and analyzed for the presence of REIC DNA (621-bp fragment) using PCR. The treatment administered in group 4 was as follows: intravenous injection via tail vein of $2.5 \times 10^{8}$ vp of Ad-REIC in $100 \mu 1$ vehicles.

after a dose of $2.5 \times 10^{8} \mathrm{vp}$ was injected into the prostate of the mice (group 2), vector Ad-REIC DNA was detected in the prostate (injection site), with trace amounts found in the brain, kidney, colon, and urinary bladder. Bands of vector DNA were also detected in the prostate on days 7 and 28. On day 1, the group that received the higher dose of $2.0 \times 10^{10} \mathrm{vp}$ of Ad-REIC (group 3) showed detectable levels of vector DNA in the lung, heart, liver, pancreas, spleen, kidney, stomach, colon, urinary bladder, prostate (injection site), testis, femoris muscle, and adrenal gland. On days 1, 7 and 28, the most pronounced levels of vector DNA were observed in the colon, urinary bladder, and prostate. On day 28, vector Ad-REIC DNA was still detectable in the colon, urinary bladder, and prostate.

The intravenous injection of Ad-REIC at a dose of $2.5 \times 10^{8}$ vp (group 4) resulted in detectable levels of vector Ad-REIC DNA in the liver, spleen, colon, and whole blood on day 1 . No detectable DNA vectors were present by day 7. A summary of the biodistribution of the Ad-REIC vectors following intraprostatic and intravenous administration is shown in Table I.

Toxicological examinations. As a preliminary experiment, the expression of REIC protein following intraprostatic Ad-REIC injection was examined in normal mouse prostate using western blot analysis. On day 3, an elevated expression of intraprostatic REIC protein, in comparison to the control treatment, was verified (Fig. 5A). In all of the experiments, the physical appearance, activity, and mortality of the mice (groups 1-4) were evaluated daily. No adverse findings or signs of toxicity were noted and no animal deaths occurred in this study. At necropsy, no inflammation-related findings, such as the presence of abscesses or abundant ascites, were detected in any of the animals.

To examine the toxicology of the Ad-REIC treatment, histological analyses were completed on sections of the brain, lung, thymus, heart, liver, pancreas, spleen, kidney, stomach, colon, urinary bladder, dorsolateral prostate (injection site in groups 1-3), testis, femoris muscle, adrenal gland, and whole
Table I. A summary of the biodistribution of the Ad-REIC vectors following intraprostatic or intravenous administration.

\begin{tabular}{lccc}
\hline & $\begin{array}{c}\text { Intraprostate } \\
(\text { lower dose })\end{array}$ & $\begin{array}{c}\text { Intraprostate } \\
\text { (higher dose) }\end{array}$ & Intravenous \\
\hline Brain & + & - & - \\
Lung & - & + & - \\
Thymus & - & + & - \\
Heart & - & + & - \\
Liver & - & + & + \\
Pancreas & - & + & - \\
Spleen & - & + & - \\
Kidney & + & + & - \\
Stomach & - & + & - \\
Colon & + & + & - \\
Urinary bladder & + & + & - \\
Prostate & + & + & - \\
(injected site) & & & + \\
Testis & - & + & - \\
Femoris muscle & - & + & - \\
Adrenal gland & - & + & - \\
Whole blood & - & + & - \\
\hline
\end{tabular}

blood. We first analyzed the local effects of Ad-REIC injection using a histopathological analysis of the dorsolateral prostate, the vector injection site. In the Ad-REIC injected prostate of group 3 , infiltrations of inflammatory cells were observed in the interstitial areas on day 1; however, no such infiltrations were observed on day 7 (Fig. 5B). Similar findings were also observed in the Ad-LacZ injected prostate; however, we were 

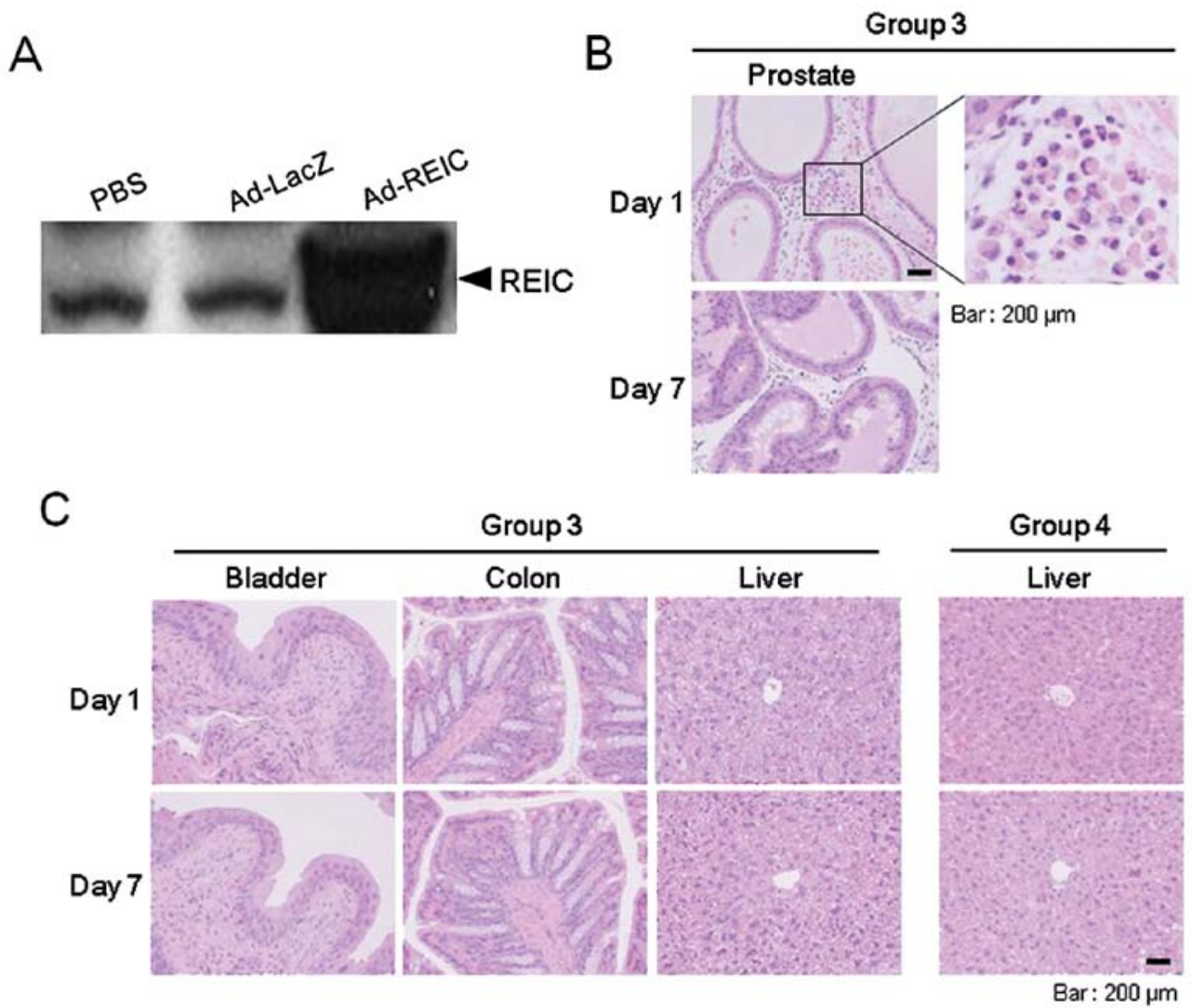

Figure 5. (A) REIC protein expression following intraprostatic Ad-REIC injection was examined in normal mouse prostate using western blot analysis. On day 3 , an elevated expression of intraprostatic REIC protein, compared to that observed in the control group, was verified. Total protein (10 $\mu \mathrm{g})$ were loaded in each lane. (B) To examine the local effects of Ad-REIC treatment, a histological analysis of the dorsolateral prostate (vector injection site) was performed. The tissue was fixed in formalin and the sections were stained with hematoxylin and eosin to analyze any histopathological and cytotoxic changes. The selected area (framed square) is magnified in the inset panel and indicates the presence of an infiltration of inflammatory cells in the interstitial area observed on day 1 following the intraprostatic injection of Ad-REIC (group 3). On day 7 following the Ad-REIC injection, the infiltration of inflammatory cells in this area was no longer observed. (C) The systemic toxicology of both intraprostatic (group 3) and intravenous (group 4) Ad-REIC treatment were examined using histological analyses. Various organs were analyzed and the histology of the urinary bladder, colon, and liver are shown as representative samples. The PCR bands of vector DNA were relatively strong and Ad-REIC indicated a tendency to disseminate in these organs. No significant histological damage or definite toxicity were observed in any of the examined organs at any time-point.

unable to detect any histological effects in the prostatic sections of the PBS treated group (group 1) (data not shown). Since the biodistribution of the Ad-REIC vectors following intraprostatic injection with the high dose (group 3) was observed to be widespread, we next investigated whether systemic toxicity resulted from Ad-REIC treatment in this group. As for tissues other than the prostate, no histopathological abnormalities were observed in the sections obtained from any treatment group at any time-point (Fig. 5C).

The liver is the primary target organ for adenoviral vectors following intravenous administration (19). We therefore examined the liver tissue of group 4. None of these mice showed any histopathological liver abnormalities, such as cytotoxicity or inflammation, during the experiment (Fig. 5C).

\section{Discussion}

The adenoviral vectors carrying the human REIC/Dkk-3 gene (Ad-REIC) selectively induce apoptosis in prostate cancer cells and other cancer cells through the activation of c-Jun-NH2-kinase (JNK) and c-Jun $(3,4,11)$. It is also reported that the adenovirusmediated expression of the REIC/Dkk-3 gene efficiently induces endoplasmic reticulum (ER) stress-mediated apoptosis in a manner specific to cancer cells $(4,20,21)$. The present study demonstrated that Ad-REIC treatment induces significant apoptosis in human prostate cancer cells and hepatoma cell lines; however, Ad-REIC treatment does not induce apoptosis in normal human cells. Therefore, the adenoviral vector agent, Ad-REIC, appears to be effective against human cancer cells and is attractive in terms of cancer-specific targeting. In the experiment evaluating the biodistribution of Ad-REIC vectors, we also examined the in vivo toxicity of the vectors using a non-tumorbearing mouse model. The aim was to examine the safety of Ad-REIC vectors and to ensure that overexpression of the REIC/ Dkk3 gene via intraprostatic injection does not result in systemic toxicity. The experiment was also designed as a preclinical study to support the future clinical use of Ad-REIC. No deaths or signs of toxicity or unusual behavior were observed in the treated mice in any group.

In this study, we used two doses $\left(2.5 \times 10^{8} \mathrm{vp}\right.$ and $\left.2.0 \times 10^{10} \mathrm{vp}\right)$ for the Ad-REIC treatment in mice. In the clinical trial planned for the patients with prostate cancer, intraprostatic injection of $1.0 \times 10^{12}$ viral particles (vp) of Ad-REIC was scheduled for one treatment. In this study, the lower dose (a dose of $2.5 \times 10^{8} \mathrm{vp}$ administered via intraprostatic or tail vein injection in groups 2 and 4 , respectively) was calculated based on the comparison of 
mouse and human body weights ( $25 \mathrm{~g}$ versus $100 \mathrm{~kg}$ ). In this experiment, the equivalent of a $1 \times 10^{12} \mathrm{vp}$ human dose/prostate was considered to be a $2.5 \times 10^{8} \mathrm{vp}$ dose in a mouse. The selection of the higher dose (group 3 ) was based on previous clinical studies of prostate cancer gene therapy in which adenovirus-mediated gene delivery was performed using a dose between $10^{10}$ and $10^{11}$ viral particles/prostate (13-15). The higher dose of $2.0 \times 10^{10} \mathrm{vp}$ is 80 -fold higher than the lower dose of $2.5 \times 10^{8} \mathrm{vp}$ which is equivalent to the highest human dose of $1 \times 10^{12} \mathrm{vp} /$ prostate anticipated to be used in the planned clinical protocol.

This study demonstrates that Ad-REIC injected into the prostate of mice disseminates to various tissues, even at the lower dose of $2.5 \times 10^{8} \mathrm{vp}$. In the organs of the mice in group 3, which were treated with the higher dose of $2.0 \times 10^{10} \mathrm{vp}$, the PCR bands of vector DNA were found to be stronger in the widespread organs, including the prostate. These findings suggest that Ad-REIC injected into the prostate passes into the general circulation and disseminates to various tissues, leading to the sporadic appearance of vector sequences in other distant organs. At the higher dose, vector Ad-REIC DNA was still detectable in the colon, urinary bladder, and prostate on day 28 . The colon and urinary bladder are located near the prostate and this may explain the tendency of Ad-REIC to disseminate to these organs after intraprostatic administration. On the other hand, in the intravenous injection via the tail vein group (group 4), Ad-REIC vectors tended to disseminate to the liver and spleen on day 1 , and vector DNA disappeared from all organs by day 28. Since the liver and spleen are organs of the reticuloendothelial system, intravenously injected Ad-REIC might be trapped more frequently in these organs. These results support the idea that the biodistribution and clearance time of Ad-REIC are dependent on the dose and route of vector administration.

Based on these biodistribution studies, we demonstrated that Ad-REIC is widely distributed in the bodies of mice after intraprostatic or intravenous vector administration. At the dose of $2.0 \times 10^{10} \mathrm{vp}$ used in group 3, residual DNA from Ad-REIC was detected in various organs and the injected prostate until day 28. A dose of $2.0 \times 10^{10} \mathrm{vp}$ is very high relative to the body weight of a mouse. Even at the high dose used in group 3, no significant histological damage or definite toxicity were observed in any organs examined at any time-point. Regarding the inflammatory cells infiltrated in the prostatic interstitial spaces in the mice in group 3, this finding was also observed in the mice in the Ad-LacZ treatment group. It seems that adenoviral vectors are directly involved in the inflammatory reactions. Across the multiple therapeutic and pharmacological studies previously conducted in mice in our laboratory, no overt signs of gross toxicity or significant findings that would raise concerns regarding unanticipated toxicological effects of Ad-REIC were observed $(3,4,8,9)$. Therefore, even in clinical situations, the risk of significant adverse effects and toxicity to other organs after intraprostatic Ad-REIC administration might be limited.

Our results show that adenoviral vectors encoding the REIC/Dkk-3 gene have no significant toxicity when injected into the prostate of normal mice at the indicated doses. It seems that Ad-REIC and its gene product, REIC protein, do not induce systemic adverse effects under these conditions. Adenovirusmediated in situ REIC/Dkk-3 gene therapy is considered to be safe for use as a treatment for human prostate cancer. We believe that the present study provides valuable information for future clinical trials with Ad-REIC and other adenovirus-mediated gene therapies used to treat human prostate cancer.

\section{Acknowledgements}

This study was supported by a scientific research grant (KAKENHI 23390382) from the Ministry of Education, Culture, Sports, Science, and Technology of Japan.

\section{References}

1. Tsuji T, Miyazaki M, Sakaguchi M, Inoue Y and Namba M: A REIC gene shows downregulation in human immortalized cells and human tumor-derived cell lines. Biochem Biophys Res Commun 268: 20-24, 2000.

2. Hsieh SY, Hsieh PS, Chiu CT and Chen WY: Dickkopf-3/REIC functions as a suppressor gene of tumor growth. Oncogene 23: 9183-9189, 2004.

3. Abarzua F, Sakaguchi M, Takaishi M, Nasu Y, Kurose K, Ebara S, Miyazaki M, Namba M, Kumon H and Huh NH: Adenovirusmediated overexpression of REIC/Dkk-3 selectively induces apoptosis in human prostate cancer cells through activation of c-Jun-NH2-kinase. Cancer Res 65: 9617-9622, 2005.

4. Kashiwakura Y, Ochiai K, Watanabe M, Abarzua F, Sakaguchi M, Takaoka M, Tanimoto R, Nasu Y, Huh NH and Kumon H: Downregulation of inhibition of differentiation-1 via activation of activating transcription factor 3 and Smad regulates REIC/ Dickkopf-3-induced apoptosis. Cancer Res 68: 8333-8341, 2008.

5. Zhang K, Watanabe M, Kashiwakura Y, Li SA, Edamura K, Huang P, Yamaguchi K, Nasu Y, Kobayashi Y, Sakaguchi M, et al: Expression pattern of REIC/Dkk-3 in various cell types and the implications of the soluble form in prostatic acinar development. Int J Oncol 37: 1495-1501, 2010.

6. Mao B, Wu W, Davidson G, Marhold J, Li M, Mechler BM, Delius H, Hoppe D, Stannek P, Walter C, et al: Kremen proteins are Dickkopf receptors that regulate Wnt/beta-catenin signaling. Nature 417: 664-667, 2002.

7. Abarzua F, Sakaguchi M, Tanimoto R, Sonegawa H, Li DW, Edamura K, Kobayashi T, Watanabe M, Kashiwakura Y, Kaku H, et al: Heat shock proteins play a crucial role in tumor-specific apoptosis by REIC/Dkk-3. Int J Mol Med 20: 37-43, 2007.

8. Edamura K, Nasu Y, Takaishi M, Kobayashi T, Abarzua F, Sakaguchi M, Kashiwakura Y, Ebara S, Saika T, Watanabe M, et al: Adenovirus-mediated REIC/Dkk-3 gene transfer inhibits tumor growth and metastasis in an orthotopic prostate cancer model. Cancer Gene Ther 14: 765-772, 2007.

9. Watanabe M, Kashiwakura Y, Huang P, Ochiai K, Futami J, Li SA, Takaoka M, Nasu Y, Sakaguchi M, Huh NH and Kumon H: Immunological aspects of REIC/Dkk-3 in monocyte differentiation and tumor regression. Int J Oncol 34: 657-663, 2009.

10. Tanimoto R, Abarzua F, Sakaguchi M, Takaishi M, Nasu Y, Kumon $\mathrm{H}$ and Huh NH: REIC/Dkk-3 as a potential gene therapeutic agent against human testicular cancer. Int J Mol Med 19: 363-368, 2007.

11. Kawasaki K, Watanabe M, Sakaguchi M, Ogasawara Y, Ochiai K, Nasu Y, Doihara H, Kashiwakura Y, Huh NH, Kumon H and Date H: REIC/Dkk-3 overexpression downregulates P-glycoprotein in multidrug-resistant MCF7/ADR cells and induces apoptosis in breast cancer. Cancer Gene Ther 16: 65-72, 2008.

12. Jin Y, Murata H, Sakaguchi M, Kataoka K, Watanabe M, Nasu Y, Kumon $\mathrm{H}$ and Huh NH: Partial sensitization of human bladder cancer cells to a gene-therapeutic adenovirus carrying REIC/Dkk-3 by downregulation of BRPK/PINK1. Oncol Rep 27: 695-699, 2012.

13. Freytag SO, Khil M, Stricker H, Peabody J, Menon M, DePeraltaVenturina M, Nafziger D, Pegg J, Paielli D, Brown S, et al: Phase I study of replication-competent adenovirus-mediated double suicide gene therapy for the treatment of locally recurrent prostate cancer. Cancer Res 62: 4968-4976, 2002.

14. Kubo H, Gardner TA, Wada Y, Koeneman KS, Gotoh A, Yang L, Kao C, Lim SD, Amin MB, Yang H, et al: Phase I dose escalation clinical trial of adenovirus vector carrying osteocalcin promoterdriven herpes simplex virus thymidine kinase in localized and metastatic hormone-refractory prostate cancer. Hum Gene Ther 14: 227-241, 2003. 
15. van der Linden RR, Haagmans BL, Mongiat-Artus P, van Doornum GJ, Kraaij R, Kadmon D, Aguilar-Cordova E, Osterhaus AD, van der Kwast TH and Bangma CH: Virus specific immune responses after human neoadjuvant adenovirus-mediated suicide gene therapy for prostate cancer. Eur Urol 48: 153-161, 2005.

16. Su C, Cao H, Tan S, Huang Y, Jia X, Jiang L, Wang K, Chen Y, Long J, Liu X, et al: Toxicology profiles of a novel p53-armed replication-competent oncolytic adenovirus in rodents, felids, and nonhuman primates. Toxicol Sci 106: 242-250, 2008

17. Belloc F, Dumain P, Boisseau MR, Jalloustre C, Reiffers J, Bernard P and Lacombe F: A flow cytometric method using Hoechst 33342 and propidium iodide for simultaneous cell cycle analysis and apoptosis determination in unfixed cells. Cytometry 17: 59-65, 1994.

18. Maciorowski Z, Delic J, Padoy E, Klijanienko J, Dubray B, Cosset JM, Dumont J, Magdelénat H and Vielh P: Comparative analysis of apoptosis measured by Hoechst and flow cytometry in non-Hodgkin's lymphomas. Cytometry 32: 44-50, 1998.
19. Muruve DA, Barnes MJ, Stillman IE and Libermann TA: Adenoviral gene therapy leads to rapid induction of multiple chemokines and acute neutrophil-dependent hepatic injury in vivo. Hum Gene Ther 10: 965-976, 1999.

20. Sakaguchi M, Kataoka K, Abarzua F, Tanimoto R, Watanabe M, Murata H, Than SS, Kurose K, Kashiwakura Y, Ochiai K, et al: Overexpression of REIC/Dkk-3 in normal fibroblasts suppresses tumor growth via induction of interleukin-7. J Biol Chem 284: 14236-14244, 2009.

21. Ochiai K, Watanabe M, Ueki H, Huang P, Fujii Y, Nasu Y, Noguchi H, Hirata T, Sakaguchi M, Huh NH, Kashiwakura Y, Kaku $\mathrm{H}$ and Kumon H: Tumor suppressor REIC/Dkk-3 interacts with the dynein light chain, Tctex-1. Biochem Biophys Res Commun 412: 391-395, 2011. 